\title{
Durchschnittskostenvergleich vs. Tarifcontrolling
}

\section{David Hofstetter}

Dr. iur., Rechtsanwalt

Die Wirtschaftlichkeit der Leistungserbringung wird in Arztpraxen überwiegend mittels des statistischen Durchschnittskostenvergleichs geprüft. In jüngerer Zeit hat die von tarifsuisse AG verwendete Methode des Tarifcontrollings an Bedeutung gewonnen. Im Rahmen des Tarifcontrollings werden Rechnungen von Leistungserbringern mittels Software auf eine auffällige Verrechnung bestimmter Tarifpositionen hin überprüft, wobei auffällig meint, dass ein Leistungserbringer bestimmte Tarifpositionen häufiger als andere Leistungserbringer verrechnet. Mit Blick auf die zugrundeliegende Methodik und weitere Aspekte wie die Verwirkung der Rückforderungsansprüche der Krankenversicherer wirft das Tarifcontrolling Fragen auf, die bis anhin von Rechtsmittelinstanzen nicht verbindlich geklärt wurden.

Nach Art. 56 Abs. 1 KVG muss sich der Leistungserbringer in seinen Leistungen auf das Mass beschränken, das im Interesse der Versicherten liegt und für den Behandlungszweck erforderlich ist. Für Leistungen, die über dieses Mass hinausgehen, kann die Vergütung verweigert oder eine dem Leistungserbringer zu Unrecht bezahlte Vergütung zurückgefordert werden (Art. 56 Abs. 2 KVG).

Die Wirtschaftlichkeit der Leistungserbringung wird in der Praxis überwiegend mittels des statistischen Durchschnittskostenvergleichs (DKV) geprüft. Im Rahmen des DKV werden die durchschnittlichen

\section{Résumé}

Dans les cabinets médicaux, la rentabilité de la prestation est essentiellement mesurée sur la base de comparaisons statistiques des coûts moyens. Plus récemment, la méthode tarifcontrolling utilisée par tarifsuisse SA a gagné de l'importance. Dans le cadre du tarifcontrolling, un logiciel vérifie que les factures des prestataires ne présentent pas d'incohérence au niveau de certaines positions tarifaires, c'est-à-dire contrôlent si un prestataire ne facture pas certaines positions tarifaires plus souvent que les autres professionnels. Au regard de la méthode de base, ainsi que d'autres aspects comme la péremption de la prétention en restitution des assureurs-maladie, le tarifcontrolling soulève des questions qui n'ont pas encore été résolues de façon définitive par les juridictions de recours.
Kosten, die ein Arzt (der besseren Lesbarkeit halber wird in vorliegendem Beitrag ausschliesslich die männliche Form verwendet) in einer bestimmten Leistungsperiode pro Behandlungsfall generiert hat, mit

Die Wirtschaftlichkeit der Leistungserbringung wird in der Praxis überwiegend mittels des statistischen Durchschnittskostenvergleichs (DKV) geprüft.

dem Fallkostendurchschnitt einer Gruppe von Leistungserbringern verglichen, deren Praxen annähernd gleiche Leistungsbedingungen wie diejenige des geprüften Arztes aufweisen. Überschreitet der statistische Mittelwert eines Arztes die Schwelle von 120 bis 130 Indexpunkten (bei den 20 bis 30 über 100 hinausgehenden Indexpunkten handelt es sich um den sog. Toleranzzuschlag [1]), so ist praxisgemäss der volle Beweis unwirtschaftlicher Behandlung erbracht, sofern die Überschreitung nicht mit Praxisbesonderheiten gerechtfertigt werden kann [2]. Neben der Anwendung des Durchschnittskostenvergleichs kann das Behandlungsverhalten eines Arztes auch systematisch auf seine Wirtschaftlichkeit überprüft werden, indem während einer bestimmten Periode sämtliche Abrechnungen geprüft werden und somit bei jedem einzelnen Patienten kontrolliert wird, ob er wirtschaftlich behandelt wurde (sog. analytische Methode). Aufgrund des 
erheblichen Aufwands dieser Methode findet sie in der Praxis - zumindest bei Arztpraxen - kaum Anwendung. Neben der soeben beschriebenen «klassischen» Wirtschaftlichkeitskontrolle mittels DKV hat die Rechnungskontrolle, das sog. Tarifcontrolling, in jüngerer Zeit an Bedeutung gewonnen. Beim Tarifcontrolling wird nicht mittels statistischer Methodik die Wirtschaftlichkeit eines Leistungserbringers untersucht, sondern eine einzelfallbezogene Überprüfung der Rechnungsstellung des Leistungserbringers zulasten der Krankenversicherer durchgeführt. Verantwortlich für das Tarifcontrolling ist die tarifsuisse AG, eine Tochtergesellschaft von santésuisse, dem Verband der

\section{Beim Tarifcontrolling wird eine einzelfall- bezogene Überprüfung der Rechnungsstellung des Leistungserbringers zulasten der Kranken- versicherer durchgeführt.}

Schweizerischen Krankenversicherer. Tarifsuisse AG umschreibt ihre diesbezügliche Zielsetzung wie folgt [3]: «Das Ziel des Tarifcontrolling ist es, Leistungserbringer zu erkennen, welche Tarife missbräuchlich anwenden oder deren Rechnungen anderweitige Unregelmässigkeiten aufweisen und damit die Wirtschaftlichkeitsgebote verletzen. Dabei wird die Tarifanwendung (Pattern) durch den Leistungserbringer gegenüber seiner Vergleichsgruppe analysiert. Können Abweichungen nicht plausibel erklärt werden, wird eine Rückzahlungsforderung gestellt.»

Einerseits prüft tarifsuisse AG in der Praxis die sog. qualitative Dignität der abrechnenden Ärzte, mithin deren Aus-, Fort- und Weiterbildungsdiplome insbesondere in den vom Besitzstand betroffenen Bereichen. Andererseits werden einzelne aus Sicht von tarifsuisse AG «auffällige» Tarifpositionen beanstandet. Auffällig meint in vorliegendem Zusammenhang, dass diese Tarifpositionen häufiger als bei anderen Ärzten angewendet wurden.

Das Tarifcontrolling unterscheidet sich in seiner Ausrichtung wesentlich von der einleitend beschriebenen Wirtschaftlichkeitskontrolle mittels DKV, obwohl der Vorwurf an sich ebenfalls in einer Verletzung des Wirtschaftlichkeitsgebotes begründet liegt. Das Bundesgericht umschreibt den Inhalt der Rechnungskontrolle wie folgt [4]: «Von der Wirtschaftlichkeitskontrolle $\mathrm{zu}$ unterscheiden ist die von den Krankenversicherern durchzuführende Rechnungskontrolle, bei welcher es vor allem darum geht, die Übereinstimmung der einzelnen Positionen der Honorarrechnungen mit den tarifvertraglichen Vereinbarungen sowie den für bestimmte Therapien gesetzlich umschriebenen Vorgaben zu prüfen [...]. Die Rech- nungskontrolle kann Elemente der Wirtschaftlichkeitsprüfung enthalten, so etwa, wenn die korrekte Tarifanwendung zur Diskussion steht [...]. Darüber hinaus kann sich die Frage stellen, ob in Rechnung gestellte Leistungen überhaupt erbracht worden sind, und ob allenfalls eine betrügerische Rechnungsstellung und damit ein strafbares Verhalten vorliegt.» Werden teurere Tarifpositionen als die medizinisch gebotenen fakturiert oder werden Tarifpositionen auf unzulässige Weise kumuliert abgerechnet, wird gemäss bundesgerichtlicher Rechtsprechung das Mass des Erforderlichen im Sinne von Art. 56 Abs. 1 KVG ebenfalls überschritten, so dass auch in diesem Fall der Tatbestand der Überarztung erfüllt sein kann [5]. Beim Tarifcontrolling haben die Krankenversicherer bzw. hat tarifsuisse AG somit einzelne Positionen der von den Ärzten erstellten Honorarrechnungen zu prüfen. Die Realität sieht jedoch - zumindest im vorprozessualen Stadium - anders aus. Konkret begründet tarifsuisse AG die Vorwürfe an die Ärzte betreffend unrichtige Anwendung von Tarifpositionen jeweils standardmässig damit, dass ein Arzt eine bestimmte Tarifposition im Vergleich zu einem von tarifsuisse AG herbeigezogenen Referenzkollektiv anderer Ärzte zu häufig angewendet und damit zu häufig verrechnet habe. Dies erinnert in der Vorgehensweise stark an den DKV, ist aber im Rahmen des Tarifcontrollings aufgrund des Überprüfungsgegenstandes nicht angezeigt. Zu überprüfen sind konkrete Rechnungen oder konkrete einzelne Rechnungspositionen. Über einen Vergleich mit einem wie auch immer zusammengesetzten Referenzkollektiv anderer Ärzte kann gerade nicht festgestellt werden, ob die einzelnen Rechnungen bzw. einzelne Positionen der Rechnung eines Leistungserbringers korrekt sind. Vielmehr hat tarifsuisse AG konkrete einzelne Rechnungen zu beanstanden, um die Vorwürfe gegenüber den angegangenen Ärzten zu untermauern.

\section{$\mathrm{Zu}$ überprüfen sind konkrete Rechnungen oder konkrete einzelne Rechnungspositionen.}

Man kann sich gar fragen, ob in diesem Verhalten eines Organs der mittelbaren Staatsverwaltung, wie es die Krankenversicherer sind, nicht ein Verstoss gegen das verfassungsrechtlich verankerte Gebot des Verhaltens nach Treu und Glauben erblickt werden muss (Art. 5 Abs. 3 und Art. 9 Bundesverfassung). Denn ohne entsprechende Kenntnisse oder Hilfestellungen wird wohl manch ein Arzt geneigt sein, der von tarifsuisse AG gestellten Forderung nachzugeben und somit eine Rückforderung zu begleichen, die auf einer methodisch unzureichenden Basis beruht. 
Eine weitere Fragestellung des Tarifcontrollings betrifft den Beginn der relativen einjährigen Verwirkungsfrist nach Art. 25 Abs. 2 ATSG. Während bei den auf dem DKV basierenden Wirtschaftlichkeitsverfahren die relative einjährige Verwirkungsfrist mit Kenntnis der hierfür massgebenden Rechnungsstellerstatistik durch die Krankenversicherer zu laufen beginnt und sich somit zuverlässig feststellen lässt [6], ist beim Tarifcontrolling eine Prüfung des Einzelfalls angezeigt. Es gilt, dass die relative Verwirkungsfrist zu laufen beginnt, sobald der Krankenversicherer vom Bestehen eines Rückforderungsanspruchs Kenntnis hat oder bei zumutbarer Aufmerksamkeit haben müsste [7]. Da die Krankenversicherer die Pflicht trifft, die Rechnungen der Leistungserbringer vor der Ausrichtung der von ihnen geleisteten Vergütung zu prüfen, beginnt die einjährige relative Verwirkungsfrist meines Erachtens spätestens im Zeitpunkt der Begleichung der vom Leistungserbringer geforderten Vergütung durch den Krankenversicherer. Dies bedeutet zugleich, dass für von den Krankenversicherern ausgerichtete Vergütungen, welche mehr als ein Jahr zurückliegen, eine Rückforderung verwirkt ist. Immerhin wird dieser Auffassung seitens der Krankenversicherer entgegengehalten, dass es sich bei der Begleichung von Leistungsabrechnungen um ein

Die relative Verwirkungsfrist beginnt zu laufen, sobald der Krankenversicherer vom Bestehen eines Rückforderungsanspruchs Kenntnis hat oder bei zumutbarer Aufmerksamkeit haben müsste.

(millionenfaches) Massengeschäft handelt und eine Überprüfung der einzelnen Rechnungen somit nicht zumutbar ist, so dass auch die einjährige Frist nicht bereits mit Begleichung der Rechnung zu laufen beginnt, sondern erst zu demjenigen Zeitpunkt, in welchem eine konkrete Prüfung des Abrechnungsverhaltens des einzelnen Leistungserbringers vorgenommen wurde.

Mit welcher konkreten Handlung tarifsuisse AG die Verwirkungsfrist unterbrechen kann, hängt davon ab, ob im betreffenden Kanton ein obligatorisches Schlichtungsverfahren durchlaufen werden muss. Besteht ein solches obligatorisches Schlichtungsverfahren, wird die Verwirkungsfrist durch die fristgerechte Anrufung der zuständigen Instanz gewahrt. Besteht kein obligatorisches Schlichtungsverfahren und muss direkt
Klage beim hierfür zuständigen Gericht erhoben werden, so genügt es für die Wahrung der Verwirkungsfrist, wenn die Krankenversicherer die Rückforderung gegenüber dem Leistungserbringer «in geeigneter Form» geltend machen [8]. Meines Erachtens muss ein derartiges Begehren zumindest den Anspruch gegenüber dem Leistungserbringer beziffern und eine Aufforderung zu dessen Begleichung enthalten, um als fristwahrende Handlung zu qualifizieren.

\section{Besteht ein obligatorisches Schlichtungs-} verfahren, wird die Verwirkungsfrist durch die fristgerechte Anrufung der zuständigen Instanz gewahrt.

Schliesslich ist auch fraglich, ob das Vorgehen von tarifsuisse AG im Rahmen des Tarifcontrollings nicht in den therapeutischen Ermessensspielraum der behandelnden Ärzte eingreift. Klar scheint zu sein, dass anhand eines im Rahmen der Rechnungskontrolle unzulässigen Vergleichs mit einem Referenzkollektiv anderer Ärzte mit Blick auf die Anwendung bestimmter Tarifpositionen keine Aussage dazu möglich ist, ob die Behandlung des Patienten im Einzelfall medizinisch indiziert und angemessen war oder nicht. Der Vorwurf, alleine die häufigere Anwendung einer bestimmten Position belege Unwirtschaftlichkeit, liegt somit in einem Spannungsverhältnis zum therapeutischen Ermessen des Arztes. Für den solcherart angegangenen Arzt ist denn auch eine wirksame Verteidigung unmöglich, weiss er doch nicht, welche Rechnungen seitens tarifsuisse AG konkret beanstandet werden. Nur eine konkrete Beanstandung würde es dem behandelnden Arzt erst ermöglichen, den Vorwurf der Überarztung mit medizinischen Argumenten zu widerlegen. Diese und andere Fragen des Tarifcontrollings harren meines Erachtens nach wie vor einer verbindlichen Klärung.

\footnotetext{
Literatur

1 BGE $137 \mathrm{~V}$ 43, E. 2.2

2 Gebhard Eugster, in: Schweizerisches Bundesverwaltungsrecht Band XIV, Soziale Sicherheit, 3. Auflage, Basel 2016, S. 677 N 879.

3 www.tarifsuisse.ch/de/leisungsmanagement-und-dienste/ tarifcontrolling

4 BGer Urteil K 124/O3, E. 6.1.2.

5 BGer Urteil K 116/03, E. 4.2.

6 BGer Urteil 9C 393/2007, E. 4.3

7 BGer Urteil K 70/06, E. 5.1 (nicht publ. in BGE 133 V 579).

8 BGE 133 V 579, E. 4.3.5
} 